TIPA. Travaux interdisciplinaires sur la parole et le langage

28 | 2012

Linguistique expérimentale

\title{
La conjugaison du verbe en début de scolarisation
}

Alexandra Marquis, Phaedra Royle, Laura Gonnerman et Susan Rvachew

\section{OpenEdition}

Journals

Édition électronique

URL : http://journals.openedition.org/tipa/201

DOI : 10.4000/tipa.201

ISSN : 2264-7082

Éditeur

Laboratoire Parole et Langage

\section{Référence électronique}

Alexandra Marquis, Phaedra Royle, Laura Gonnerman et Susan Rvachew, « La conjugaison du verbe en début de scolarisation », TIPA. Travaux interdisciplinaires sur la parole et le langage [En ligne], 28 | 2012, mis en ligne le 29 octobre 2012, consulté le 30 avril 2019. URL : http:// journals.openedition.org/tipa/201; DOI : 10.4000/tipa.201

Ce document a été généré automatiquement le 30 avril 2019

\section{cc) $(1) \ominus$}

La revue TIPA. Travaux interdisciplinaires sur la parole et le langage est mise à disposition selon les termes de la licence Creative Commons Attribution - Pas d'Utilisation Commerciale - Pas de Modification 4.0 International. 


\title{
La conjugaison du verbe en début de scolarisation
}

\author{
Alexandra Marquis, Phaedra Royle, Laura Gonnerman et Susan Rvachew
}

\section{Cadre théorique}

\subsection{Acquisition}

1 L'acquisition productive du langage nécessite la maîtrise de plusieurs phénomènes linguistiques, dont l'identification des structures et des éléments morphologiques du mot (ex. racine+flexion). Cette identification morphologique est particulièrement importante dans la conjugaison des verbes, qui occupent un point central dans la structure de la phrase (Bassano, 2010). Les enfants francophones sont sensibles à la productivité morphologique, mais sont également sensibles à la régularité et à la fréquence d'apparition des schèmes verbaux français (Royle et al. 2012). Chez le nourrisson, la reconnaissance de la racine d'un verbe survient entre huit et onze mois (Marquis \& Shi, 2008) tout comme la capacité de relier la racine d'un verbe à une de ses formes conjuguées (Marquis \& Shi, 2009). Dès l'âge de trois ans, les enfants démontrent l'utilisation de principes morphologiques qui distinguent les groupes de conjugaison lors de la production des verbes (ex. Royle, 2007). La recherche sur l'acquisition des verbes en langage spontané nous indique que les enfants francophones (entre 21 et 47 mois) produisent des formes conjuguées à l'impératif, au futur proche, à l'imparfait et au passé composé (Thordardottir, 2005). De plus, les formes produites par les enfants francophones sont généralement grammaticales: les verbes sont conjugués selon la personne, le nombre et le temps.

\subsection{Modèles théoriques}

2 Il existe des modèles psycholinguistiques (basés sur l'anglais) dans lesquels les verbes sont décrits de façon dichotomique, réguliers versus irréguliers (voir par ex. Pinker, 1999). Les verbes réguliers se conjuguent en suivant un schème morphologique régulier (ex. call, 
call+ing, call+ed) et productif qui peut être recruté pour leur production (ou compréhension), tandis que les verbes irréguliers semblent se conjuguer sans concaténation morphologique (ex. sing, sang, sung) et doivent être mémorisés et emmagasinés pour leur production adéquate. Pour les langues latines (ex. l'espagnol, l'italien ou le français), la conjugaison des verbes est mieux décrite en termes de distinction tripartite des groupes du verbe (voir par ex. Clahsen et al., 2002, pour l'espagnol ; Royle et al., 2012, pour le français; Say \& Clahsen, 2002, pour l'italien). En français par exemple, les verbes sont traditionnellement divisés en trois groupes principaux : les verbes du premier groupe (se terminant en -er à l'infinitif), les verbes du second groupe (se terminant en -ir à l'infinitif) et les verbes irréguliers ${ }^{1}$. Cependant, des sous-groupes de régularité se manifestent parmi les trois groupes. Certains de ces sousgroupes semblent emprunter, notamment pour le passé composé, des schèmes de conjugaison chez les verbes réguliers (ex. les irréguliers en -é, aller - allé /ale/) et sousréguliers (ex. les irréguliers en $-i$, prendre - pris / $\mathrm{pвi} /$ ), tandis que d'autres se conjuguent de façon irrégulière (ex., ouvrir - ouvert /uvEs/). Parmi les verbes du troisième groupe, un sous-groupe particulier émerge : les verbes dont le participe passé se termine en -u (ex. voir $-v u$ ). Ce sous-groupe renferme des verbes parmi les plus fréquents de la langue tels que voir, boire et vouloir. Étant donné que la fréquence d'occurrence d'un verbe peut influencer sa maîtrise chez l'enfant (voir par ex. Royle, 2007), il s'avère important d'étudier aussi la connaissance des schèmes réguliers et sous-réguliers qui apparaissent fréquemment dans la langue. Or, le manque de distinction de ces verbes sous-réguliers et irréguliers peut mener à une compréhension superficielle des capacités à conjuguer les verbes chez les enfants.

\subsection{Effets de fréquence}

3 Même si les verbes du premier groupe composent la majorité des verbes français (de $85 \%$ à $90 \%$ de tous les verbes, Bescherelle, 2006), les effets de fréquence de type et d'occurrence (en anglais type et token frequency) affectent l'exposition à tous les groupes de conjugaison. Selon le corpus de langage spontané de deux enfants francophones de deux ans de Guillaume (1927 [1973]), les verbes du premier groupe représentent $76 \%$ des types de verbes, alors qu'ils ne constituent que $36 \%$ des occurrences. Les verbes du second groupe (verbes réguliers et irréguliers) incluent $6 \%$ des types et $11 \%$ des occurrences, tandis que les verbes du troisième groupe (tous irréguliers) comptent $18 \%$ des types et $58 \%$ des occurrences. Les verbes irréguliers semblent donc être très présents dans la langue de l'enfant. Nicoladis et al. (2007) ont vérifié l'importance de la fréquence des formes du passé composé pour l'acquisition des verbes, en analysant des corpus de littérature enfantine et de discours dirigé vers l'enfant. Leur investigation dévoile que $63 \%$ des types de verbes au passé composé et $51 \%$ des occurrences sont du premier groupe. Royle et al. (2012) ont confirmé les données de Guillaume (1927[1973]) avec un corpus plus récent (2005-2008) de 12 enfants québécois âgés de 36 à 45 mois. De plus, une analyse des formes au passé composé démontre la prédominance des schèmes du premier groupe (61\% des types et $47 \%$ des occurrences) pour cette conjugaison, avec une représentativité mitigée du second groupe ( $17 \%$ des types et $27 \%$ des occurrences, si on y inclut les verbes irréguliers ayant un participe passé en $-i$ ) comparativement aux verbes dont le participe passé se termine en - $u$ ( $9 \%$ des types $5 \%$ des occurrences), et aux verbes avec une forme non morphologique tel mourir-mort (11\% des types et $19 \%$ des occurrences). Ces analyses démontrent que les formes du passé composé incluent une 
grande variété de formes, avec une prédominance du premier groupe. Une étude vérifiant les capacités de production des enfants de ces quatre groupes de verbes permettrait de dévoiler la sensibilité des enfants à la fréquence des schèmes morphologiques des verbes français.

\subsection{Trouble spécifique du langage (TSL)}

L'enseignement du français en milieu scolaire requiert, outre la compétence à écrire, l'enseignement de la conjugaison des verbes. Afin de faciliter cet enseignement, il est nécessaire de bien connaître les principes grammaticaux (c'est-à-dire, la grammaire implicite) présents chez l'enfant avant l'entrée à l'école, les difficultés rencontrées par les enfants, et les moyens qu'ils utilisent pour surmonter leurs difficultés de conjugaison. De plus, un certain nombre d'enfants présente des troubles du langage qui auront des impacts directs sur leur réussite scolaire. En particulier, les enfants présentant un trouble spécifique du langage (TSL), une dyslexie ou une dysorthographie peuvent être en difficulté en milieu scolaire. L'un des principaux problèmes rencontrés par les enfants avec un TSL est la conjugaison des verbes (ex. Jakubowicz \& Nash, 2001 ; Paradis \& Crago, 2001 ; Royle \& Thordardottir, 2008). Or, même pour les enfants sans trouble de langage, trop peu d'études traitent de la nature des processus impliqués dans l'encodage des paradigmes verbaux. Il apparaît que les enfants sans trouble établissent précocement une sensibilité aux schèmes morphologiques des verbes français. Jakubowicz \& Nash (2001), Paradis \& Crago, (2001), et Royle \& Thordardottir (2008) ont démontré que les enfants avec TSL éprouvent des difficultés avec les flexions verbales du français comparativement aux groupes contrôles. Lorsque l'on compare la production de verbes induits, les enfants âgés de 3-4 ans avec TSL produisent significativement moins le passé composé contrairement aux enfants sans trouble de langage (Royle \& Thordardottir, 2008). Dans une étude précédente de Royle (2007), portant sur la conjugaison chez quinze enfants à développement typique et âgés de 3 à 4 ans et demi, environ $10 \%$ des erreurs commises étaient des erreurs de surgénéralisation, où la forme cible était fléchie en empruntant un schéma régulier (ex. *il a voulé) ou sous-régulier (ex. *il a ouvri), phénomène rare chez les enfants TSL. Les erreurs de surgénéralisation indiquent que les enfants ne font pas que copier ce que les adultes produisent, mais qu'ils génèrent des schèmes de conjugaison permettant de produire les formes peu fréquentes ou irrégulières. Par contre, l'étude de Royle (2007) ne contrôle pas spécifiquement les stimuli verbaux selon la classification tripartite présentée ci-dessus, et n'étudie pas non plus la sensibilité à la régularité phonologique présente dans les formes se terminant en -u.

\subsection{Productivité}

$5 \quad$ L'indice de productivité du passé composé peut s'avérer essentiel afin de déterminer les effets de la fréquence et de la régularité sur l'acquisition de la conjugaison des verbes français. Le participe passé est très fréquent dans le langage chez l'enfant et représente pour les verbes d'action environ $16 \%$ des formes verbales produites spontanément entre 14 et 36 mois (corpus de Pauline, Bassano, 2010). Hiriarteborde (1973) a développé une tâche de production induite du passé composé où la production induite des verbes réguliers fréquents et moins fréquents était comparée au discours spontané des mêmes enfants. À trois ans et demi, les petits Français produisaient correctement le passé composé, mais seulement si l'on retrouvait au moins quatre différents types de passé 
composé dans leur discours spontané. Les enfants québécois démontrent la même capacité de produire le passé composé de verbes connus vers trois ans et demi et de verbes inconnus vers quatre ans (Royle, 2007). Kresh (2008) a étudié le traitement du participe passé en français à l'aide d'une tâche de production induite chez des enfants de six et huit ans. Dans cette étude, en plus des traditionnels groupes de verbes dont le participe se termine en -é, en -i et les irréguliers (ex. souffrir-souffert), Kresh a évalué le sous-groupe des verbes irréguliers dont le participe se termine en - $u$ (ex. paraitre-paru). Les résultats de cette étude indiquent des effets de fréquence pour tous les items sauf ceux en - $u$, et des effets paradoxaux pour les formes irrégulières : en effet, on observe un renversement de l'effet de fréquence pour les verbes les moins fréquents qui sont mieux produits que ceux de fréquence moyenne. Cependant, seuls des items de moyenne ou basse fréquence ont été testés dans cette étude. On peut aussi se questionner sur la représentativité des mesures de fréquence utilisées (fréquence du Lemme en texte écrit dans MANULEX, Lété et al., 2004), étant donné les résultats rapportés pour les formes irrégulières. En prenant pour acquis que les enfants sont sensibles aux effets de fréquence du type, une étude plus approfondie de ces effets sur les verbes de haute fréquence, tout en contrôlant pour les sous-groupes de conjugaison des verbes français apparaît nécessaire, afin de mieux comprendre le traitement des différentes formes verbales. Dans la présente étude, nous reprendrons les groupes de verbes en -é, en - $i$, en - $u$ et irréguliers (IR) étudiés par Kresh (2008), mais en n'utilisant que des formes de fréquence relativement haute.

\subsection{Problématique}

6 Afin de réussir l'acquisition adéquate des paradigmes verbaux, les enfants doivent dans un premier temps déterminer l'existence des différents groupes de conjugaison, malgré la haute représentativité d'un seul schéma (les verbes en -er) dans le corpus. En parallèle, ils devront développer des règles d'application de ces schèmes (c'est-à-dire connaître la classe verbale à laquelle une forme appartient). Dans la présente étude, nous voulons évaluer la maîtrise du passé composé chez les enfants de 6 et 7 ans, en utilisant des verbes avec les terminaisons du participe passé suivants; soit en -é (ex. cacher-caché), en -i (ex. finir-fini), en -u (ex. mordre-mordu) et les irréguliers autres (ex. ouvrir-ouvert). Selon le Programme de formation de l'école québécoise du Ministère de l'enseignement, des loisirs et du sport du Québec (le MELS), les enfants n'ont pas encore appris à distinguer ni à écrire ces formes ${ }^{2}$. Nous pouvons donc étudier les compétences linguistiques de ces enfants sans grande interférence avec la scolarisation. Ceci nous permettra de développer un modèle de la représentation du verbe chez l'enfant en début de scolarisation.

\section{Hypothèses}

Dans un premier temps, on s'attend à obtenir un effet d'âge qui distinguerait les enfants plus âgés des enfants plus jeunes. On prévoit également d'obtenir un meilleur taux de réussite selon la régularité et la fréquence du paradigme : les verbes réguliers en -é seront mieux réussis que les verbes en $-i$, qui, eux, seront mieux réussis que les verbes en - $u$, qui à leur tour seront mieux réussis que les verbes irréguliers autres. Étant donné les résultats de Royle (2007) démontrant des sur-régularisations en -i chez des enfants de 4 ans - les verbes en $-i$ devraient être bien maîtrisés - Il est probable que cela se retrouve au niveau 
des verbes du premier groupe. Les verbes irréguliers, en $-\mathfrak{u}$ et autres, seront moins bien réussis que les verbes réguliers et sous-réguliers dû à leur manque de productivité. Les verbes en $-u$ seront mieux réussis que les verbes irréguliers autres, malgré leur absence de productivité, en raison de la présence d'une régularité morphophonologique en fin de mot (-u) (voir Albright, 2002 pour une discussion de la fiabilité (reliability) morphologique, c'est-à-dire le potentiel qu'une marque morphologique donnée puisse être utilisée dans un environnement phonologique déterminé), et de la fréquence d'item de ces formes. Finalement, pour les verbes irréguliers autres, aussi improductifs, et étant des types uniques, nous pensons qu'aucun effet de fréquence de type ne peut favoriser leur apprentissage : ils devraient, par conséquent, être les plus difficiles à produire malgré leur grande fréquence d'item.

\section{Expérience}

\subsection{Méthodologie}

\subsubsection{Participants}

8 Vingt-deux enfants de maternelle (13 garçons et 9 filles) âgés de 6 ans $(M=6 ; 1, E T=0,31)$ et treize enfants de première année ( 9 garçons et 4 filles) âgés de 7 ans $(M=7 ; 8 E T=0,32)$ tous unilingues francophones de la grande région de Laval, Québec ont participé à cette étude. ${ }^{3}$ La collecte de données s'est déroulée aux mois de mai et juin. Les enfants étaient donc tous en fin d'année scolaire. Les enfants de 6 ans terminaient la maternelle et les enfants de 7 ans terminaient, quant à eux, la première année. Les enfants participaient à une étude plus large visant à développer un outil de dépistage de la dysorthographie en français (Rvachew et al., 2011-2013), le protocole a été approuvé par les comités d'éthique à la recherche des facultés de médecine des Universités de McGill et de Montréal. Les parents ou tuteurs des enfants ont signé un formulaire de consentement permettant à l'enfant de participer à l'étude. Les enfants pouvaient se retirer de l'étude à tout moment. Nous avons également amassé des informations démographiques, d'exposition à l'écrit, de santé et de langage par le biais d'une entrevue téléphonique avec les parents. Selon les informations obtenues, aucun des enfants de notre groupe n'avait de signes de troubles neuronaux, cognitifs ou autres pouvant affecter le développement de son langage.

\subsubsection{Procédure}

9 Nous avons créé une tâche de production induite de verbes sur tablette interactive ${ }^{4}$ à plate-forme Android. Une application en code source libre (Open Source) développée pour la tâche permettait de présenter des images créées par une artiste professionnelle et illustrant les verbes induits. L'application, consiste à simuler un livre d'histoires imagées avec lequel l'enfant est amené à compléter de courtes histoires en répondant à des questions posées par l'évaluateur. L'application enregistrait toutes les réponses verbales des enfants en plus d'une série d'informations telles que la date et l'heure de l'expérimentation ainsi que le temps écoulé pour le visionnement de chacune des images.

Les enfants ont tous été testés individuellement à l'école durant les heures de classe dans un local de l'établissement scolaire réservé pour l'étude, pour une durée approximative de 30 à 45 minutes. Durant l'expérimentation, l'enfant avait en main la tablette présentant les images et pouvait observer les images à sa guise. L'expérimentatrice lisait 
les phrases avec les verbes cibles visant à induire la production du passé composé en français. Cette méthode a été adaptée à partir de celle utilisée avec des enfants francophones beaucoup plus jeunes par Royle (2007) et Royle \& Thordardottir (2008) et d'enfants plus âgés par Kresh (2008) (voir aussi Berko, 1958 pour son travail séminal sur l'utilisation de la méthodologie de production induite avec des enfants anglophones). L'expérimentatrice passait à l'image suivante si l'enfant ne répondait pas (c'est-à-dire jusqu'à ce que le silence devienne inconfortable, soit après environ 20 secondes) suivant la lecture du script. Peu importe sa production, l'enfant était encouragé pour ses efforts avant de passer à l'image suivante. Un exemple du script est donné en (1).

11 (1) Image : Une fille cachant ses poupées sous une boîte

- Marie va cacher ses poupées.

- Marie cache toujours ses poupées.

- Qu'est-ce qu'elle a fait hier Marie?

L'expérience débutait avec les quatre stimuli d'entraînement, un pour chaque groupe de verbes étudié. Pour les stimuli d'entrainement seulement, la bonne réponse était donnée à l'enfant s'il ne pouvait produire la réponse. Le script donnait des indices sur le paradigme de conjugaison en fournissant la forme du verbe au présent (ex. cache) et à l'infinitif, soit dans une forme avec un auxiliaire (ex. va cacher) ou avec un verbe demandant un complément à l'infinitif (ex. aime cacher).

\subsection{Stimuli}

13 Nous avons utilisé quatre groupes de sept verbes chacun $(N=28): 7$ verbes avec un participe passé en -é (ex. cacher-caché), 7 verbes avec un participe passé en - $i$ (ex. finir-fini), 7 verbes avec un participe passé en - $u$ (ex. mordre-mordu) et 7 verbes avec un participe passé autre ou non paradigmatique (ex. ouvrir-ouvert). Les groupes de verbes étaient équilibrés sur des mesures de fréquence (d'après les bases de données LEXIQUE et MANULEX pour les 6-7 ans), voir les Annexes 1 et 2. Les fréquences de forme et de lemme ne diffèrent pas statistiquement (tous les $p>1$ ). Les groupes de verbes ont également été appariés pour le nombre de phonèmes, le nombre de syllabes, le nombre de voisins orthographiques et le nombre de voisins phonologiques (tous les $p>.1$ ). Avant la collecte des données, nous avons fait évaluer la plausibilité des phrases porteuses par des adultes locuteurs natifs du français $(N=7)$. Toutes les phrases ont été jugées plausibles en français à $96 \%(E T=1.07)$.

L'ordre de présentation des stimuli était le même pour tous les enfants. La présentation des images a préalablement été pseudo-randomisée pour qu'au maximum deux verbes d'un même groupe soient présentés de façon contiguë, afin de réduire les stratégies de production d'analogies selon le modèle des stimuli précédents. Un point était accordé pour la production du passé composé complet (le groupe sujet-auxiliaire-participe, ex. ( Marie), elle a caché). Toutes les réponses ont été enregistrées et les types d'erreurs ont par la suite été compilés et analysés. De plus, une analyse d'erreurs a permis de vérifier plus à fond les stratégies utilisées par les enfants. Les types d'erreurs observés sont a) le participe produit seul (ex. bu pour il a bu : ce type d'erreur est ambigu entre l'infinitif et le participe passé pour les verbes en -é), b) l'infinitif produit seul (ex. boire pour il a bu), c) le verbe au présent (ex. il boit pour il a bu), et d) autre production (la substitution d'un autre verbe ou la production mixte du passé composé avec la forme infinitive, ex. il a terminé pour il a fini ou encore *il a ouvrir pour il a ouvert). 


\section{Résultats}

Dans toutes les analyses, la valeur d'alpha de 0,05 a été utilisée comme seuil de signification. Des analyses à mesures répétées (ANOVA) ont été faites sur les scores moyens de réponses cibles des enfants, avec le groupe de verbes comme facteur intrasujets ( $-\dot{e},-i,-u$ et autres) et le groupe d'âge comme facteur intersujets (maternelle et première année). Nous avons trouvé un effet principal de groupe de verbes $(F(3,31)=$ $63.3, p<.001)$, mais aucun effet principal de groupe d'âge $(F(1,33)=1.13, p=.295)$, ni interaction de groupe de verbes et groupe d'âge $(F(3,31)=1.26, p=.305)$, suggérant que les résultats ne diffèrent pas selon l'âge des enfants. Ces résultats sont rapportés au Tableau 1 et dans la Figure 1. Compte tenu des résultats de l'ANOVA, nous avons comparé les résultats des quatre groupes de verbes au moyen de tests post-hoc. Les verbes en -é sont significativement mieux produits que les trois autres groupes de verbes : $-e ́$ contre $-i$ $t(34)=4.35, p<.001 ;-e ́$ contre $-u t(34)=5.41, p<.001 ;-e ́$ contre autres $t(34)=4.35, p<.001$. Les verbes autres sont significativement moins bien produits que les autres groupes de verbes: autres contre $-i t(34)=7.76, p<.001$; autres contre $-u t(34)=6.54, p<.001$. Finalement, les scores moyens pour les verbes des groupes $-i$ et $-u$ ne diffèrent pas entre $\operatorname{eux} t(34)=1.58, p=.124$.

Tab. 1. Pourcentage d'énoncés réussis et écarts-types pour chacun des groupes de verbes

\begin{tabular}{|l|l|l|l|l|l|l|}
\hline & \multicolumn{5}{|l|}{ Maternelle } & \multicolumn{2}{l|}{$\begin{array}{l}\text { Première } \\
\text { année }\end{array}$} & \multicolumn{2}{l|}{$\begin{array}{l}\text { Tous les } \\
\text { enfants }\end{array}$} \\
\hline & $M$ & $E T$ & $M$ & $E T$ & $M$ & $E T$ \\
\hline$-e ́$ & 93,18 & 21,61 & 100 & 0 & 95,71 & 17,31 \\
\hline$-i$ & 69,70 & 38,36 & 73,08 & 31,58 & 70,95 & 35,55 \\
\hline$-u$ & 62,88 & 34,09 & 67,95 & 38,16 & 64,76 & 35,19 \\
\hline autre & 27,27 & 20,28 & 46,15 & 25,60 & 34,29 & 23,89 \\
\hline Moyenne & 63,26 & & 71,79 & & 66,43 & \\
\hline
\end{tabular}


Fig. 1. Pourcentage de réponses cibles par groupe de verbes pour chaque groupe d'âge

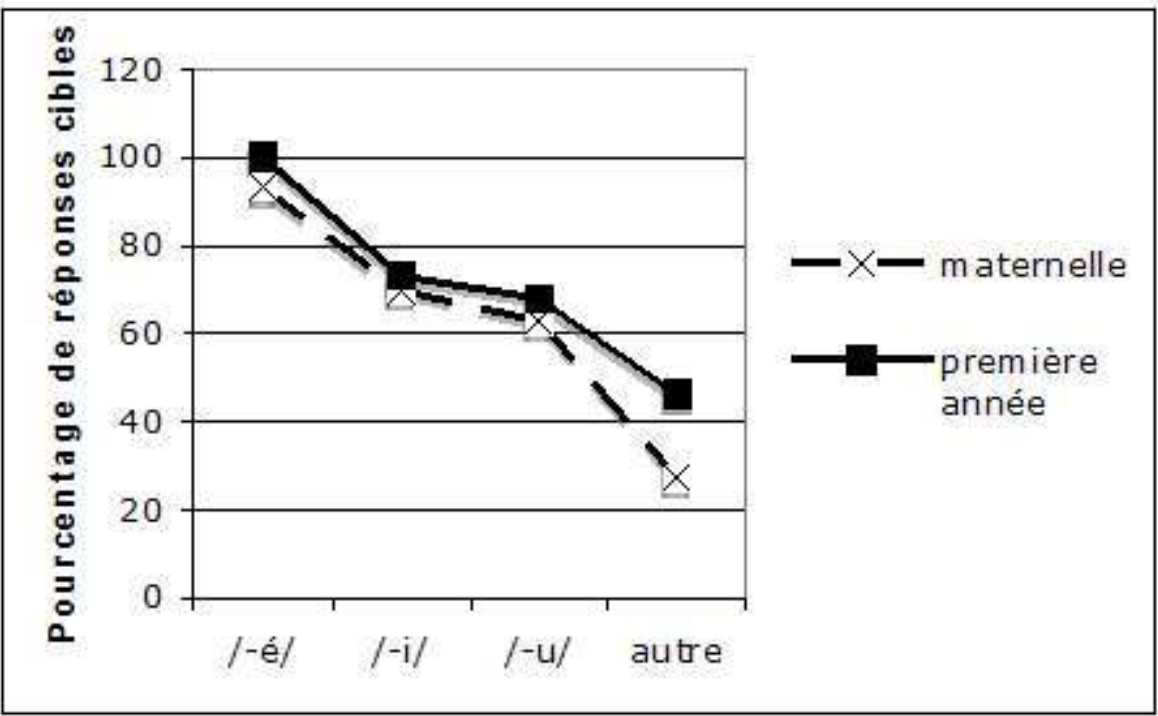

Puisque différents types d'erreurs ont été relevés dans les productions des enfants, nous avons vérifié si les erreurs se retrouvaient de façon similaire à travers les quatre groupes de verbes. Nous avons regroupé ici la production de l'infinitif, la production d'un autre verbe, la production mixte du passé composé avec la forme infinitive ou la production d'une forme surgénéralisée, car ces erreurs étaient trop peu fréquentes pour les intégrer dans des cellules individuelles dans nos analyses statistiques non paramétriques.

Des analyses Chi-carrés à deux niveaux, avec comme facteurs le groupe de verbes (quatre niveaux) et le type d'erreurs (cible, participe passé, présent de l'indicatif et autres) sur les fréquences des stratégies de réponses non-cibles, ont révélé une relation significative entre les groupes de verbes et le type d'erreurs, $X^{2}(9, N=810)=235.13, p<.001$, voir le Tableau 2 pour les détails de la répartition des erreurs réelles et attendues. Nous observons une différence dans la distribution des erreurs entre le groupe des verbes en -é et les trois autres groupes de verbes. Pour les verbes en -é, les erreurs consistent presque exclusivement en la production de la forme ambiguë du participe passé ou de l'infinitif [radical+e] à $25,2 \%$. Pour les trois autres groupes de verbes, les erreurs se situent au niveau du groupement autres (23,6\% pour les verbes en $-i ; 29,2 \%$ pour les verbes en $-u$; $58,4 \%$ pour les verbes autres).

Tab. 2. Fréquence des types d'erreurs par groupes de verbes (-é, -i, -u et autres) pour tous les enfants

\begin{tabular}{|l|l|l|l|l|l|}
\hline \multicolumn{4}{|l|}{ Production } & \\
\hline $\begin{array}{l}\text { Type } \\
\text { participe }\end{array}$ & Cible & Participe & Présent & Autre & Total \\
\hline$-e ́$ & & & & & \\
\hline Observé & $148(70,5 \%)$ & $53(25,2 \%)$ & $7(3,3 \%)$ & $2(1 \%)$ & 210 \\
\hline
\end{tabular}




\begin{tabular}{|c|c|c|c|c|c|}
\hline Attendu & 126,3 & 18,4 & 8,0 & 57,3 & \\
\hline \multicolumn{6}{|l|}{$-i$} \\
\hline Observé & $140(67,3 \%)$ & $9(4,3 \%)$ & $10(4,8 \%)$ & $49(23,6 \%)$ & 208 \\
\hline Attendu & 125,1 & 18,2 & 8,0 & 56,8 & \\
\hline \multicolumn{6}{|l|}{$-u$} \\
\hline Observé & $129(63,9 \%)$ & $7(3,5 \%)$ & $7(3,5 \%)$ & $59(29,2 \%)$ & 202 \\
\hline Attendu & 121,4 & 17,7 & 7,7 & 55,1 & \\
\hline \multicolumn{6}{|l|}{ Autre } \\
\hline Observé & $70(36,8 \%)$ & $2(1,1 \%)$ & $7(3,7 \%)$ & $111(58,4 \%)$ & 190 \\
\hline Attendu & 114,2 & 16,7 & 7,3 & 51,8 & \\
\hline
\end{tabular}

Nous avons voulu vérifier plus avant la répartition des erreurs dans les groupes de verbes n'étant pas du premier groupe, entre autres la production de l'infinitif et le participe passé. Nous avons donc comparé les groupes de verbes $-i,-u$ et autres avec les types d'erreurs (cible, participe passé, infinitif, présent de l'indicatif et autre production ${ }^{5}$ ). L'analyse Chi-carré révèle encore une fois une relation significative entre les groupes de verbes et le type d'erreurs $X^{2}(8, N=610)=114.34, p<.001$, voir le Tableau 3. Cette dernière analyse indique que la production de l'infinitif constitue entre 16,5 et 19,3\% des erreurs de production pour tous les verbes (à l'exclusion du premier groupe) tandis que la production du participe seul est peu présente. Nous pouvons également voir que les enfants produisent $44 \%$ d'autres productions pour les verbes à schème non paradigmatique. En résumé, pour toutes les analyses, nous pouvons remarquer l'absence de différence entre les verbes du groupe $-i$ et les verbes du groupe $-u$. Nous remarquons également une maîtrise significativement plus élevée pour les verbes du groupe -é.

Tab. 3. Fréquence des types d'erreurs par groupes de verbes (-i, - $u$ et autres) pour tous les enfants

\begin{tabular}{|c|c|c|c|c|c|c|}
\hline & \multicolumn{5}{|l|}{ Production } & \multirow[b]{2}{*}{ Total } \\
\hline & Cible & Participe & Infinitif & Présent & Autre & \\
\hline$-i$ & & & & & & \\
\hline Observé & $140(67,3 \%)$ & $9(4,3 \%)$ & $35(16,8 \%)$ & $10(4,8 \%)$ & $14(6,7 \%)$ & 208 \\
\hline Attendu & 115,6 & 6,1 & 36,5 & 8,2 & 41,6 & \\
\hline$-u$ & & & & & & \\
\hline
\end{tabular}




\begin{tabular}{|l|l|l|l|l|l|l|}
\hline Observé & $129(63,9 \%)$ & $7(3,5 \%)$ & $39(19,3 \%)$ & $7(3,5 \%)$ & $20(9,9 \%)$ & 202 \\
\hline Attendu & 112,3 & 6,0 & 35,4 & 7,9 & 40,4 & \\
\hline Autre & & & & & & \\
\hline Observé & $70(35,0 \%)$ & $2(1,0 \%)$ & $33(16,5 \%)$ & $7(3,5 \%)$ & $88(44,0 \%)$ & 200 \\
\hline Attendu & 111,1 & 5,9 & 35,1 & 7,9 & 40 & \\
\hline
\end{tabular}

\section{Discussion}

La maîtrise productive des processus morphologiques de la langue permet entre autres de pouvoir reconnaître des mots nouvellement rencontrés. Cette capacité est nécessaire aux enfants qui sont en train de bâtir le lexique de leur langue. La présente étude a tenté de démontrer la pertinence d'étudier les effets de fréquence des groupes et sous-groupes de régularité des verbes français. Nous avons testé la capacité des enfants à produire des verbes réguliers, sous-réguliers et irréguliers en vérifiant aussi la maitrise de verbes qui sont généralement considérés comme irréguliers: les verbes dont le participe passé se termine en $-u$ (ex. voir-vu). Nous avions émis comme hypothèse que les enfants plus jeunes éprouveraient plus de difficultés à produire le passé composé que les enfants plus âgés. Cette hypothèse est infirmée, les plus jeunes comme les plus vieux ont réussi à produire avec un succès similaire les formes induites. Il y a deux interprétations possibles de ce résultat. La première est que notre mesure n'est pas assez sensible pour révéler un changement des capacités linguistiques dans ces groupes d'âge (une autre étude pourrait par exemple vérifier la capacité des enfants à produire le participe passé sans égard à la syntaxe), et la seconde est qu'il n'y a pas de différences entre les enfants âgés de 6 et 7 ans dans la représentation de la morphologie verbale. Des études futures pourront répondre à ces questions. Nous avions également prévu d'obtenir un effet gradient de réussite selon la régularité et la fréquence du paradigme $\left(-e^{-}>-i>-u>\right.$ autres). Cette hypothèse est en partie confirmée puisque les verbes réguliers en -é ont été significativement mieux réussis que les verbes des trois autres groupes, et les verbes irréguliers autres significativement moins bien réussis en général. Cependant, il était surprenant de constater qu'il n'y avait aucune différence entre les verbes en -i et en - $u$. Ces derniers sont traditionnellement considérés comme irréguliers et tardivement maîtrisés. Les présentes données suggèrent que ces verbes peuvent être relativement bien appris par les enfants, peut-être dû à la présence d'une sous-régularité au niveau de la flexion (voyelle finale /i/ ou /y/). Nos données diffèrent des résultats obtenus par Kresh (2008), qui n'avait pas obtenu d'effets de fréquence pour les verbes en - $u$, et a montré un effet gradient de réussite sur les différents groupes de verbes peu fréquents et de fréquence moyenne.

La présente étude évoque plutôt une distinction tripartite des schèmes réguliers, sousréguliers et irréguliers des verbes fréquents en français, ce qui est différent de l'approche dichotomique de schèmes réguliers versus irréguliers proposée par Kresh (2008) et Nicoladis et al. (2007). Les verbes sous-réguliers en - $i$ et en - $u$ (ces derniers généralement catégorisés comme étant irréguliers) sont produits avec un succès similaire chez les 
enfants de 6 et 7 ans. Les enfants vus dans cette étude n'ont pas encore reçu d'enseignement explicite des règles de conjugaison des verbes. Ils nous informent donc des schèmes de conjugaison établis en majorité par exposition implicite.

21 Notre analyse révèle que non seulement les enfants réussissent à produire correctement ces verbes, mais qu'il est impossible de distinguer statistiquement les capacités des enfants à produire les sous-groupes du groupe des verbes en -i et en -u. Nous estimons que c'est l'interaction entre la fréquence d'occurrence et la fiabilité morphophonologique qui a permis aux enfants de maîtriser ces conjugaisons (qui sont peu représentatives en termes de types selon les données de Royle et al. 2012). Ces résultats devraient servir de point d'ancrage aux modèles psycholinguistiques de l'acquisition des conjugaisons des verbes français.

Cette étude a été menée avec l'appui financier du FQRSC (2011-ER-144359, Rvachew et al.) et du CRSH (410-2009-0256, Royle et al.).

Nous remercions Gina Cook du iLangage.ca pour l'application Android, Catherine Lamirande pour la cueillette et la transcription des données, l'école (anonyme) nous ayant accueillis ainsi que tous les enfants qui ont participé au projet.

\section{BIBLIOGRAPHIE}

Albright, A. (2002) Islands of reliability for regular morphology: Evidence from Italian, Language, 78, p. 684-709.

Bassano, D. (2010) L'acquisition des verbes en français : un exemple de l'interface lexique / grammaire, Synergies France, 6, p. 27-39.

Berko, J. (1958). The child's learning of English morphology, Word, 14, p. 150-177.

Bescherelle, L. N. (2006) Le nouveau Bescherelle 1. L'art de conjuguer; dictionnaire de 12000 verbes, Montréal : Éditions Hurtubise HMH.

Clahsen, H., Aveledo, F., Roca, I. (2002) The development of regular and irregular verb inflection in Spanish child language, Journal of Child Language, 29, p. 591-622.

Guillaume, P. (1927[1973]) Le développement des éléments formels dans le langage de l'enfant, Journal de Psychologie, 24, p. 203-229.

Hiriarteborde, A. (1973) Sur la généralisation de quelques marques grammaticales dans le langage d'enfants de 3 ans 1/2, Études de Linguistique Appliquée, 9, p. 101-124.

Jakubowicz, C. \& Nash, L. (2001) Functional categories and syntactic oprerations in (ab)normal language acquisition, Brain and Language, 77, p. 321-339.

Kresh, S. (2008) L'acquisition et le traitement de la morphologie du participe passé en français, Mémoire de maîtrise, Université du Québec à Montréal.

Lété, B., Sprenger-Charolles, L., Colé, P. (2004) MANULEX: A grade-level lexical database from French elementary-school readers, Behavior Research Methods, Instruments, \& Computers, 36, p. 156-166.

TIPA. Travaux interdisciplinaires sur la parole et le langage, 28 | 2012 
Marquis, A. \& Shi, R. (2008) Segmentation of verb forms in preverbal infants, Journal of the Acoustical Society of America, 123, 4, EL p. 105-110.

Marquis, A. \& Shi, R. (2009) The recognition of verb roots \& bound morphemes when vowel alternations are at play. Article publié en ligne dans J. Chandlee, M. Franchini, S. Lord et M. Rheiner (Eds.), A Supplement to the Proceedings of the $33^{\text {rd }}$ Boston University Conference on Language Development.

MELS (2008a) L'éducation au Québec, Tiré de http://www.mels.gouv.qc.ca/scolaire/educqc/ systemeScolaire/

MELS (2008b) Programme de formation de l'école québécoise, Tiré de http://www.mels.gouv.qc.ca/ sections/programmeFormation/

MELS. (2008c) Programme de formation de l'école québécoise : progression des apprentissages au primaire. Section conjugaison, Tiré de http://www.mels.gouv.qc.ca/progression/francaisEns/index.asp? page $=$ conn_con

New, B., Pallier, C., Ferrand, L., Matos, R. (2001) Une base de données lexicales du français contemporain sur internet : LEXIQUE, L'Année Psychologique, 101, p. 447-462.

Nicoladis, E., Palmer, A., Marentette, P. (2007) The role of type and token frequency in using past tense morphemes correctly, Developmental Science, 10, 2, p. 237-254.

Paradis, J. \& Crago, M. (2001) The Morphosyntax of Specific Language Impairment in French: An Extended Optional Default Account, Language Acquisition, 9, p. 269-300.

Pinker, S. (1999) Words and Rules: The Ingredients of Language. New York, NY: Basic Books.

Royle, P. (2007) Variable effects of morphology and frequency on inflection patterns in French preschoolers, The Mental Lexicon Journal, 2, 1, p. 103-125.

Royle, P., Beritognolo, G., Bergeron, E. (2012) Regularity, sub-regularity and irregularity in French acquisition. Dans J. van der Auwera, T. Stolz, A. Urdze \& H. Otsuka (Eds.), Irregularity in Morphology (and Beyond). Berlin: Akademie Verlag.

Royle, P. \& Thordardottir, E. T. (2008) Elicitation of the passé composé in French preschoolers with and without language impairment, Applied Psycholinguistics, 29, p. 341-365.

Rvachew, S., Gonnerman, L., Royle, P. (2011-2013) Développement d'un outil de dépistage de la dysorthographie basé sur des compétences multiples du langage oral : un nouvel outil normé et validé pour le français québécois. FQRSC Actions concertées (2011-ER-144359).

Say, T., \& Clahsen, H. (2002) Words, rules and stems in the Italian mental lexicon. Dans S. Nooteboom, F. Weerman \& F. Wijnen (Eds.), Storage and Computation in the Language Faculty, Kluwer: Dordrecht, p. 93-129.

Thordardottir, E. T. (2005) Early lexical and syntactic development in Quebec French and English: Implications for cross-linguistic and bilingual assessment. International Journal of Language and Communication Disorders, 40, p. 243-278.

\section{ANNEXES}


Annexe A : Propriétés des quatre types de verbes utilisés et écarts-types

\begin{tabular}{|c|c|c|c|c|c|c|}
\hline $\begin{array}{l}\text { Groupe de } \\
\text { verbe }\end{array}$ & $\begin{array}{l}\text { Fréquence de } \\
\text { forme }^{\mathrm{a}}\end{array}$ & $\begin{array}{l}\text { Fréquence de } \\
\text { lemme }^{\mathrm{a}}\end{array}$ & $\begin{array}{ll}\text { Nombre de } \\
\text { Phonèmes }^{\mathrm{a}}\end{array}$ & $\begin{array}{l}\text { Nombre de } \\
\text { Syllabes }^{\mathrm{b}}\end{array}$ & $\begin{array}{l}\text { Voisins } \\
\text { ortho. }^{\mathrm{a}}\end{array}$ & $\begin{array}{l}\text { Voisins } \\
\text { phono. }\end{array}$ \\
\hline -é & $110,4(141,40)$ & $535,6(572,65)$ & $\begin{array}{l}4,17 \\
(0,41)\end{array}$ & $\begin{array}{l}2 \\
(0)\end{array}$ & $\begin{array}{l}4,83 \\
(1,72)\end{array}$ & $\begin{array}{l}17,67 \\
(8,33)\end{array}$ \\
\hline$-i$ & $74,50(104,85)$ & $255,95(218,04)$ & $\begin{array}{l}3,86 \\
(1,07)\end{array}$ & $\begin{array}{l}1,71 \\
(0,49)\end{array}$ & $\begin{array}{l}5,43 \\
(4,96)\end{array}$ & $\begin{array}{l}8,86 \\
(9,30)\end{array}$ \\
\hline$-\mathrm{u}$ & $98,04(109,11)$ & $393,95(248,92)$ & $\begin{array}{l}4,17 \\
(1,17)\end{array}$ & $\begin{array}{l}2 \\
(0,63)\end{array}$ & $\begin{array}{l}5,33 \\
(5,28)\end{array}$ & $\begin{array}{l}10,33 \\
(7,23)\end{array}$ \\
\hline autre & $61,72(114,73)$ & $231,52(330,99)$ & $\begin{array}{l}3,71 \\
(1,11)\end{array}$ & $\begin{array}{l}1,71 \\
(0,49)\end{array}$ & $\begin{array}{l}4,17 \\
(4,67)\end{array}$ & $\begin{array}{l}13,5 \\
(9,59)\end{array}$ \\
\hline $\begin{array}{l}\text { A TIRÉ DE LEXIQU } \\
\text { B STRUCTURES S } \\
\text { ANALYSES UNIV }\end{array}$ & $\begin{array}{l}\text { EW ET AL., 2001) } \\
\text { BIQUES DU FRAN } \\
\text { ES PAR TYPE DE } V\end{array}$ & $\begin{array}{l}\text { JÉBÉCoIS } \\
\text { TOUS } F(3,23), P>.1\end{array}$ & & & & \\
\hline
\end{tabular}

Annexe B : Fréquences écrites de lemme et de forme et écarts-types dans la littérature enfantine

\begin{tabular}{|c|c|c|c|c|c|c|}
\hline Groupe de verbe & Lemme $\mathrm{CP}^{\mathrm{a}}$ & Lemme $C E 1^{\mathrm{a}}$ & Lemme CP-CM2 ${ }^{\mathrm{a}}$ & $\begin{array}{l}\text { Forme } \\
\mathrm{CP}^{\mathrm{a}}\end{array}$ & Forme CE $1^{\mathrm{a}}$ & $\begin{array}{l}\text { Forme } \\
\text { CP-CM2a }\end{array}$ \\
\hline -é & $\begin{array}{l}678,83 \\
(485,00)\end{array}$ & $\begin{array}{l}780,82 \\
(773,74)\end{array}$ & $\begin{array}{l}652,04 \\
(673,42)\end{array}$ & $\begin{array}{l}84,95 \\
(76,84)\end{array}$ & $\begin{array}{l}70,64 \\
(74,27)\end{array}$ & $\begin{array}{l}70,44 \\
(75,79)\end{array}$ \\
\hline$-i$ & $\begin{array}{l}290,63 \\
(208,00)\end{array}$ & $\begin{array}{l}446,92 \\
(567,95)\end{array}$ & $\begin{array}{l}407,56 \\
(478,28)\end{array}$ & $\begin{array}{l}57,72 \\
(57,48)\end{array}$ & $\begin{array}{l}71,54 \\
111,12)\end{array}$ & $\begin{array}{l}63,35 \\
(85,47)\end{array}$ \\
\hline$-\mathrm{u}$ & $\begin{array}{l}333,94 \\
(232,32)\end{array}$ & $\begin{array}{l}390,16 \\
(394,22)\end{array}$ & $\begin{array}{l}358,06 \\
(254,40)\end{array}$ & $\begin{array}{l}69,82 \\
(108,88)\end{array}$ & $\begin{array}{l}40,33 \\
(52,52)\end{array}$ & $\begin{array}{l}52,70 \\
(60,72)\end{array}$ \\
\hline autre & $\begin{array}{l}157,03 \\
(230,96)\end{array}$ & $\begin{array}{l}128,97 \\
(163,33)\end{array}$ & $\begin{array}{l}156,68 \\
(164,71)\end{array}$ & $\begin{array}{l}21,22 \\
(25,73)\end{array}$ & $\begin{array}{l}17,18 \\
(9,20)\end{array}$ & $\begin{array}{l}23,22 \\
(14,12)\end{array}$ \\
\hline \multicolumn{7}{|c|}{$\begin{array}{l}\text { A TIRÉ DE MANULEX (LÉTÉ ET ALL, 2004) } \\
\text { CP = COURS PRÉPARATOIRE OU MATERNELLE ( } 6 \text { ANS) } \\
\text { CE1 = COURS ÉLÉMENTAIRE } 1 \text { ( } 7 \text { ANS) } \\
\text { CM2 = COURS MOYEN } 2 \text { (11 ANS) } \\
\text { ANALYSES UNIVARIÉES PAR TYPE DE VERBE, TOUS P > . }\end{array}$} \\
\hline
\end{tabular}




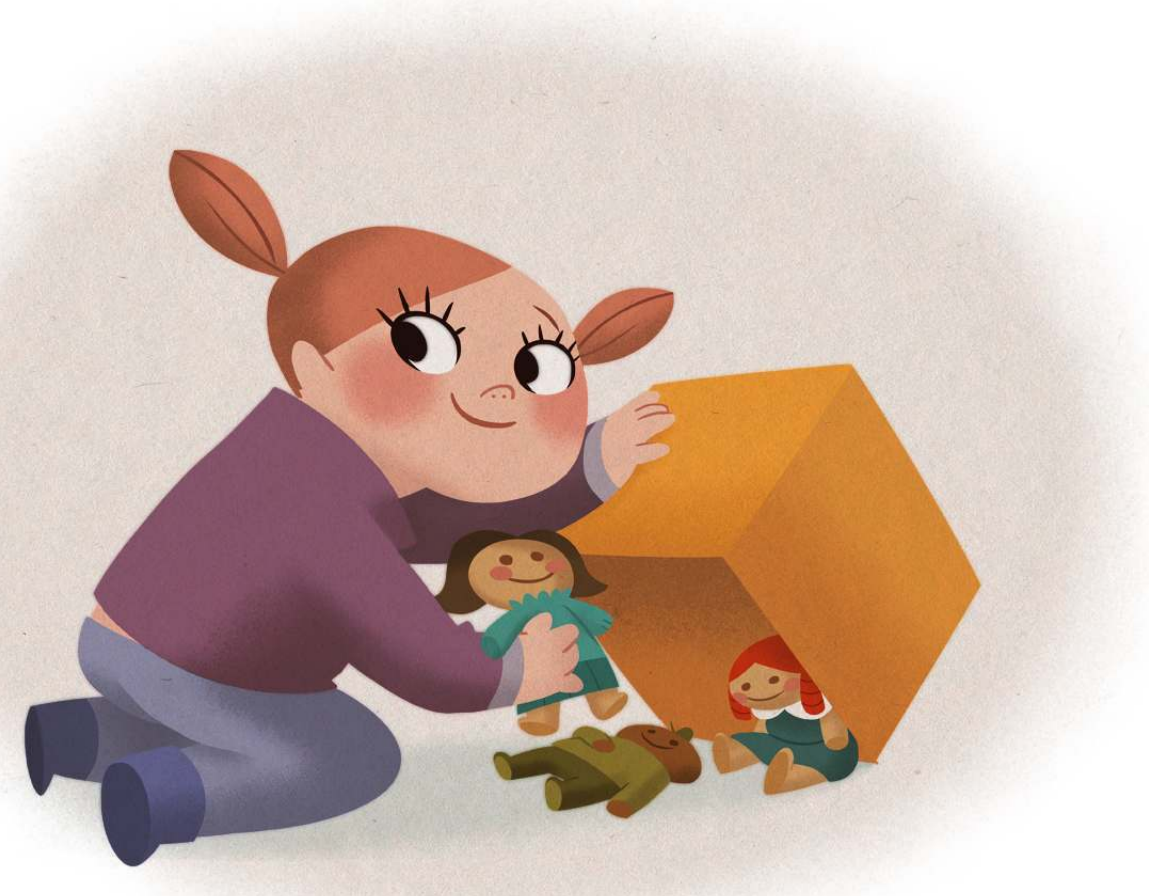

(c) Tous droits réservés Édith Lebel.

\section{NOTES}

1. Certains soutiennent par contre qu'il n'existe que deux groupes de verbes en français similairement aux modèles anglais : réguliers et irréguliers (ex. Kresh, 2008; Nicoladis et al., 2007).

2. Sur le site Web du MELS, il est indiqué que l'enfant de $1^{\text {ère }}$ et $2^{\text {ème }}$ année (premier cycle) apprend, en conjugaison, à « Mémoriser, sans découverte du système de la conjugaison, les formes verbales fréquentes à l'écrit des verbes aimer, aller, avoir, dire, être, faire à l'indicatif présent accompagnées de leur pronom de conjugaison (ex. : tu aimes; je suis; il $a$; vous faites ; ils vont) » (MELS, 2008c). Aucun de ces verbes ne fait partie de nos stimuli.

3. Dans le système d'éducation québécois, le niveau primaire compte six années de scolarité (l'âge d'admission en première année est fixé à six ans, MELS, 2008a) et la maternelle est une année facultative (l'âge d'admission en maternelle est de cinq ans, MELS, 2008b). La maternelle permet à l'enfant de socialiser : on utilise le jeu comme principale méthode d'enseignement.

4. Dans son utilisation, la tablette interactive présente plusieurs avantages : notamment elle est légère, portable, visuelle et interactive. De plus, les enfants sont friands des nouvelles technologies, ce qui assure un plus grand intérêt durant la tâche.

5. Regroupant la production d'un autre verbe, la production mixte du passé composé avec la forme infinitive ou la production d'une forme surgénéralisée. 


\section{RÉSUMÉS}

Nous avons évalué 35 enfants français québécois sur la conjugaison de verbes français réguliers, sous-réguliers et irréguliers au passé composé (en -é, -i, - -u ou autres). Une tâche de production induite a été administrée à des enfants fréquentant la maternelle ou la première année d'école. Les verbes ont été présentés, avec des images les représentant, dans des contextes à l'infinitif (ex. Marie va cacher ses poupées) et au présent (ex. Marie cache toujours ses poupées), afin d'amorcer leur paradigme de conjugaison. Les enfants devaient produire la forme cible du verbe au passé composé en répondant à la question 'Qu'est-ce qu'il/elle a fait hier ?'. Les résultats n'indiquent aucun changement dans le type et le nombre d'erreurs commises avec l'âge. Les réponses des enfants démontrent un effet de la fréquence, mais aussi de la productivité et de la prévisibilité, des différents schèmes morphologiques sur la maîtrise de la conjugaison. Les données ont des conséquences sur les modèles psycholinguistiques du traitement et de l'acquisition de la morphologie régulière et irrégulière.

We evaluated 35 Québec French children on their ability to produce regular, sub-regular, and irregular passé composé verb forms (ending in -é, $-i,-u$ or other). An elicitation task was administered to children attending preschool or first grade. Target verbs were presented, along with images representing them, in infinitive (e.g., Marie va cacher ses poupées 'Mary aux.pres. hide-inf. her dolls'= 'Mary will hide her dolls') and present tense (ex. Marie cache toujours ses poupées 'Mary hide-3s. always her dolls'= 'Mary always hides her dolls')_contexts, in order to prime the appropriate inflectional ending. Children were asked to produce target verb forms in the passé composé (perfect past) by answering the question 'What did he/she do yesterday?'. Results show no reduction of erroneous productions or error types with age. Response patterns highlight morphological pattern frequency effects, in addition to productivity and reliability effects, on children's mastery of French conjugation. These data have consequences for psycholinguistic models of regular and irregular morphology processing and acquisition.

\section{INDEX}

Keywords : verbs, conjugation group, elicitation, French, Android tablet

Mots-clés : verbes, groupe de conjugaison, production induite, français, tablette Android

\section{AUTEURS}

\section{ALEXANDRA MARQUIS}

École d'orthophonie et d'audiologie, Université de Montréal et CRMLB

alexandra.marquis@umontreal.ca 


\section{PHAEDRA ROYLE}

École d'orthophonie et d'audiologie, Université de Montréal et CRMLB phaedra.royle@umontreal.ca

\section{LAURA GONNERMAN}

CRBLM et Ecole des sciences de la communication humaine, Université McGill Laura.gonnerman@mcgill.ca

\section{SUSAN RVACHEW}

CRBLM et Ecole des sciences de la communication humaine, Université McGill susan.rvachew@mcgill.ca 